\title{
Pre-Transplant Angiotensin II Type 1 Receptor Antibodies and Anti-Endothelial Cell Antibodies Predict Graft Function and Allograft Rejection in a Low-Risk Kidney Transplantation Setting
}

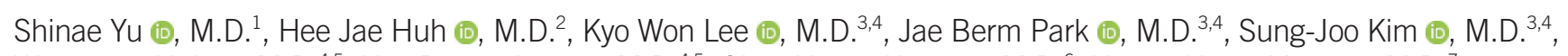

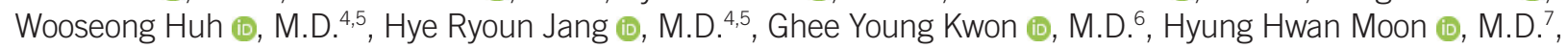
and Eun-Suk Kang $\mathbb{0}$, M.D. ${ }^{2,4}$

${ }^{1}$ Department of Laboratory Medicine, Haeundae Paik Hospital, Inje University College of Medicine, Busan, Korea; Departments of ${ }^{2}$ Laboratory Medicine and Genetics, ${ }^{3}$ Surgery, ${ }^{4}$ Organ Transplantation Center, ${ }^{5}$ Internal Medicine, ${ }^{6}$ Pathology, Samsung Medical Center, Sungkyunkwan University School of Medicine, Seoul, Korea; ${ }^{7}$ Department of Surgery Kosin University Gospel Hospital, Medical College of Kosin University, Busan, Korea

Background: Non-HLA antibodies, anti-angiotensin II type 1 receptor antibodies (antiAT1R) and anti-endothelial cell antibodies (AECA), are known to play a role in allograft rejection. We evaluated the role of both antibodies in predicting post-transplant outcomes in low-risk living donor kidney transplantation (LDKT) recipients.

Methods: In 94 consecutive LDKT recipients who were ABO compatible and negative for pre-transplant HLA donor-specific antibodies, we determined the levels of anti-AT1Rs using an enzyme-linked immunosorbent assay and the presence of AECAs using a flow cytometric endothelial cell crossmatch (ECXM) assay with pre-transplant sera. Hazard ratio (HR) was calculated to predict post-transplant outcomes.

Results: Pre-transplant anti-AT1Rs ( $\geq 11.5 \mathrm{U} / \mathrm{mL}$ ) and AECAs were observed in 36 (38.3\%) and 22 recipients (23.4\%), respectively; 11 recipients had both. Pre-transplant anti-AT1Rs were a significant risk factor for the development of acute rejection (AR) (HR 2.09; $P=0.018$ ), while a positive AECA status was associated with AR or microvascular inflammation only (HR 2.47; $P=0.004)$ throughout the follow-up period. In particular, AECA (+) recipients with $\geq 11.5 \mathrm{U} / \mathrm{mL}$ anti-AT1Rs exhibited a significant effect on creatinine and estimated glomerular filtration rate $(P<0.001 ; P=0.028)$, although the risk of AR was not significant.

Conclusions: Pre-transplant anti-AT1Rs and AECAs have independent negative effects on post-transplant outcomes in low-risk LDKT recipients. Assessment of both antibodies would be helpful in stratifying the pre-transplant immunological risk, even in low-risk LDKT recipients.

Key Words: Non-HLA antibodies, Anti- angiotensin II type 1 receptor antibodies, Anti-endothelial cell antibodies, Endothelial cell crossmatch, Kidney transplantation, Outcome, Low-risk
Received: November 13, 2019

Revision received: February 17, 2020

Accepted: March 3, 2020

\section{Corresponding author:}

Eun-Suk Kang, M.D., Ph.D.

Department of Laboratory Medicine and

Genetics, Samsung Medical Center,

Sungkyunkwan University School of

Medicine, 81 Irwon-ro, Gangnam-gu,

Seoul 06351, Korea

Tel.: +82-2-3410-2703

Fax: +82-2-3410-2719

E-mail: eskang@skku.edu 


\section{INTRODUCTION}

Human leukocyte antigen (HLA) system antigens are major barriers for the acceptance of kidney transplants. HLA donor-specific antibodies (DSA), present before or induced after kidney transplantation (KT), have been associated with hyper-acute and acute humoral rejection episodes, graft vasculopathy, graft loss, and poor long-term graft survival [1]. Appropriate donors can be chosen based on several histocompatibility assessments. However, both allograft rejections and graft failures can occur after transplantation even in HLA-identical sibling transplantations [2].

Previous studies have provided evidence for the association between non-HLA antibodies, such as angiotensin II type 1 receptor antibodies (anti-AT1Rs) and anti-endothelial cell antibodies (AECAs), and acute rejection (AR) and/or graft failure [3-13]. Indeed, the allograft endothelium is the first barrier between self and non-self in vascularized solid organ transplantations and an active target of the host immune response [14]. Anti-AT1Rs can also cause excessive activation of signal transduction pathways in the vessel endothelium, which is associated with vascular inflammatory damage [15]. AECAs activate the vascular endothelium, amplifying allo-immune responses, such as the increased expression of adhesion molecules and the production of inflammatory cytokines, which increase the degree of microvascular injury [11].

Several studies examining the significance of anti-AT1Rs or AECAs and their contribution to transplant outcomes have inevitably analyzed the antibodies in the presence of HLA antibodies because of the appearance of de novo DSA in recipients with pre-transplant non-HLA antibodies [12, 13, 16]. Considering the mechanism of action [11, 15], anti-AT1Rs or AECAs may frequently work together with DSA to exacerbate allo-immune responses in $\mathrm{KT}[7,17]$. The significance of pre-transplant nonHLA antibodies in low-risk recipients is poorly understood [16]. We aimed to investigate the significance of pre-transplant antiAT1Rs and AECAs that affect KT outcome in ABO compatible low-risk living donor kidney transplantation (LDKT) recipients without preformed DSA. In addition, we attempted to identify a correlation between serum anti-AT1R levels and AECAs.

\section{MATERIALS AND METHODS}

\section{Recipient population}

This prospective study included total 94 recipients among 201 consecutive recipients who underwent an $\mathrm{ABO}$-compatible
LDKT at Samsung Medical Center (SMC), Seoul, Korea, between January 2012 and September 2014. A negative, complement-dependent microcytotoxicity crossmatch (CDC-XM) for T and $B$ donor cells, without historic and pre-transplant HLA-DSA, was required for inclusion in this study. Furthermore, recipients who underwent desensitization owing to high panel reactive antibodies without DSA and multi-organ or combined kidney and bone marrow transplantation cases were excluded. This study was approved by the Institutional Review Board of SMC (approval number: SMC 2011-05-084), consistent with the Declaration of Helsinki, and written informed consent was obtained from all participants prior to their inclusion in the study.

\section{Measurement of anti-AT1Rs using enzyme-linked immunosorbent assay (ELISA)}

Anti-AT1R levels were measured in 123 sera using an ELISA (One Lambda Inc., Luckenwalde, Germany); 94 pre-transplant sera were acquired at the time of CDC-XM, and 29 post-transplant sera were acquired at the time of the kidney biopsy from recipients who had experienced biopsy proven rejection. AntiAT1R level in each sample was derived from a standard curve and was defined as positive when $>17 \mathrm{U} / \mathrm{mL}$, at-risk when 10 $17 \mathrm{U} / \mathrm{mL}$, and negative when $<10 \mathrm{U} / \mathrm{mL}$, according to the manufacturer's recommendations. Each run was validated with one positive and one negative control included in the kit.

\section{Detection of AECAs using flow cytometric endothelial cell crossmatch (ECXM) assay}

The presence of pre-transplant IgG and IgM antibodies against human endothelial progenitor cells (EPCs) was assessed using the XM-ONE assay (Olerup SSP AB [previously Absorber AB], Stockholm, Sweden) according to the manufacturer's instructions. A total of 94 pairs of peripheral blood samples, which were obtained from donors and recipients at the time of the final crossmatch (XM) before KT, were used for the XM-ONE assay. Donor peripheral blood mononuclear cells separated from $32 \mathrm{~mL}$ of whole blood were collected in CPT tubes (Becton Dickinson, Heidelberg, Germany) and incubated at $4^{\circ} \mathrm{C}$ for 30 minutes with paramagnetic nanobeads coated with antibodies against the Tie-2 receptor, an angiopoietin receptor, in order to isolate the EPCs. The isolated donor EPCs were incubated with recipient serum, as well as positive and negative control sera, for 30 minutes. After washing, the EPCs were incubated with fluorescein isothiocyanate-conjugated secondary antibodies against IgG and $\operatorname{lgM}$ at $4^{\circ} \mathrm{C}$ for 20 minutes. The cells were analyzed using a FACSCanto II flow cytometer and FACSDiva software v1.6 (Bec- 
ton Dickinson, San Jose, CA, USA). The cut-offs for positive EPC XM were $\geq 50$ and $\geq 80$ fluorescence channel-shift above the negative control for IgG and IgM, respectively.

\section{HLA testing}

All transplant donors and recipients were typed for HLA-A, -B, and -DRB1 using LIFECODES HLA-SSO Typing Kits (Immucor Transplant Diagnostics, Inc., Stamford, CT, USA). HLA-DQB1 was typed retrospectively only in recipients who had HLA-DQB1 antibodies pre-transplant or developed them post-transplant. Recipient HLA antibodies were evaluated as HLA class I or II using the LIFECODES LifeScreen Deluxe assay and/or the LIFECODES LSA class I and II ID Single Antigen kit (Immucor Transplant Diagnostics, Inc.). The definition of de novo HLA antibodies included DSAs against HLA-A, -B, or -DRB1 identified by single antigen identification assay and HLA-DQB1 antibodies that developed in post-transplant sera.

\section{Kidney histology}

AR included biopsy-proven AR (BPAR), according to the revised Banff 2017 classification [18], and clinically suspected AR (CSAR; $\geq 25 \%$ increase in blood creatinine from baseline, or proteinuria $>0.5 \mathrm{~g} /$ day), which improved following empirical steroid pulse therapy. Microvascular inflammation (MVI) was defined as a sum score of glomerulitis (g) and peritubular capillaritis (ptc) $\geq 2$, which is one of the three criteria for antibodymediated rejection (ABMR), according to the revised Banff 2017 classification [18]. Protocol biopsies were performed in 65 recipients at $1,3,6$, and 12 months post-transplant and indication biopsies for renal dysfunction were performed in 15 recipients. Fourteen recipients who had not been subjected to kidney biopsy during the follow-up period were considered as having no signs of clinical rejection.

\section{Data collection and statistical analysis}

All clinical data were obtained from medical records. Creatinine levels and estimated glomerular filtration rate (eGFR), calculated using the modification of diet in renal disease equation were analyzed up to three years post-KT.

Statistical analysis was performed using SAS version 9.4 (SAS Institute Inc., Cary, NC, USA). Fisher's exact tests were used for categorical variables. Wilcoxon signed-rank tests and KruskalWallis tests were used for non-parametric continuous variables. The optimal cut-off value for anti-AT1Rs was determined using a Log-rank test and Youden's index method. The repeated measurements of serum creatinine levels and eGFR at 1, 3, 6, 12,
24 , and 36 months post-transplant were analyzed using the generalized estimating equation; $P<0.05$ was considered significant. The potential risk factors of AR or MVI only within $1,3,6$, and 12 months post-transplant and during the follow-up period were analyzed using Logistic regression analysis and Cox pro-

Table 1. LDKT recipient characteristics

\begin{tabular}{|c|c|}
\hline Characteristics & Recipients $(\mathrm{N}=94)^{*}$ \\
\hline Age (yr) & $49.5(39-56)$ \\
\hline Gender, male & $57(60.6 \%)$ \\
\hline BMI & $23.1 \pm 3.8$ \\
\hline \multicolumn{2}{|l|}{ Diagnosis } \\
\hline Diabetic nephropathy & $25(29.8 \%)$ \\
\hline IgA nephropathy & $16(17.0 \%)$ \\
\hline Hypertensive nephrosclerosis & $15(16.0 \%)$ \\
\hline Glomerulonephritis & $10(10.6 \%)$ \\
\hline Other causes & $7(7.4 \%)$ \\
\hline Unknown & $18(19.2 \%)$ \\
\hline Re-transplantation & $1(1.1 \%)$ \\
\hline \multicolumn{2}{|l|}{ Induction therapy regimen } \\
\hline Anti-thymocyte globulin & $10(10.6 \%)$ \\
\hline Basiliximab & $84(89.4 \%)$ \\
\hline \multicolumn{2}{|c|}{ Maintenance regimen immunosuppressants } \\
\hline $\mathrm{CsA}+\mathrm{MMF}+\mathrm{PD}$ & $13(13.8 \%)$ \\
\hline $\mathrm{FK}+\mathrm{MMF}+\mathrm{PD}$ & $79(84.0 \%)$ \\
\hline Sirolimus or everolimus combination & $2(2.1 \%)$ \\
\hline \multicolumn{2}{|l|}{ HLA mismatches } \\
\hline Class 1 (HLA-A, -B) & $2(1-3)$ \\
\hline Class 2 (HLA-DR) & $1(0-1)$ \\
\hline \multicolumn{2}{|l|}{ CPRA } \\
\hline $0 \%$ & $72(76.6 \%)$ \\
\hline$<50 \%$ & $18(19.1 \%)$ \\
\hline$\geq 50 \%$ & $4(4.3 \%)$ \\
\hline Pre-transplant AECA (+) & $22(23.4 \%)$ \\
\hline Pre-transplant anti-AT1R levels, U/mL & $10.2 \pm 4.8$ \\
\hline AR, during F/U period & $43(45.7 \%)$ \\
\hline MVI only (g+ptc $\geq 2$ ), during $F / U$ period & $6(6.4 \%)$ \\
\hline
\end{tabular}

${ }^{*}$ Continuous variables are reported as mean \pm SD or median (interquartile range) and categorical variables are listed as total number (\%). KolmogorovSmirnov test was employed for testing normality assumption.

Abbreviations: LDKT, living donor kidney transplantation; BMI, body mass index; CsA, cyclosporine A; MMF, mycophenolate mofetil; PD, prednisolone; FK, tacrolimus; CPRA, calculated panel reactive antibodies; AECA, anti-endothelial cell antibodies; Anti-AT1R, anti- angiotensin II type 1 receptor antibodies; MVI, microvascular inflammation; g, glomerulitis; ptc, peritubular capillaritis; AR, acute rejection; F/U, follow-up. 


\section{Yu S, et al.}

Pre-transplant anti-AT1R and AECA in low-risk KT

portional-hazard regression analysis, respectively, and the proportional hazard assumption was determined using a Supremum test. Factors with $P<0.2$ in the univariate analysis were included in the multivariate analysis, and multicollinearity was determined using a variance inflation factor. $P$ and $95 \%$ confidence intervals were corrected using Bonferroni's method in cases of multiple analyses.

Table 2. Recipient characteristics and post-transplant outcomes according to anti-AT1R levels and AECAs using ECXM assay

\begin{tabular}{|c|c|c|c|c|c|c|c|}
\hline & $\begin{array}{c}\text { Anti-AT1R-negative } \\
(<10 \mathrm{U} / \mathrm{mL}) \\
(\mathrm{N}=45)^{*}\end{array}$ & $\begin{array}{c}\text { Anti-AT1R at-risk } \\
(10-17 \mathrm{U} / \mathrm{mL}) \\
(\mathrm{N}=41)^{*}\end{array}$ & $\begin{array}{c}\text { Anti-AT1R-positive } \\
(>17 \mathrm{U} / \mathrm{mL}) \\
(\mathrm{N}=8)^{*}\end{array}$ & $P$ & $\begin{array}{l}\text { AECA (-) } \\
(\mathrm{N}=72)^{*}\end{array}$ & $\begin{array}{l}\text { AECA (+) } \\
(\mathrm{N}=22)^{*}\end{array}$ & $P$ \\
\hline Age (yr) & $50(40-55)$ & $50(39-56)$ & $45.5(39.5-59.5)$ & 0.975 & $50(40-56)$ & $46(37-53)$ & 0.437 \\
\hline Gender, male & $32(71.1 \%)$ & $22(53.7 \%)$ & $3(37.5 \%)$ & 0.097 & $43(59.7 \%)$ & $14(63.6 \%)$ & 0.807 \\
\hline BMI & $23.6 \pm 3.5$ & $22.9 \pm 4.2$ & $20.7 \pm 2.4$ & 0.069 & $23.5 \pm 4.0$ & $21.6 \pm 2.9$ & 0.05 \\
\hline \multicolumn{8}{|l|}{ HLA mismatches } \\
\hline Class 1 (HLA-A, -B) & $2(1-3)$ & $2(2-3)$ & $2(1-2.5)$ & 0.683 & $2(1-3)$ & $2(2-3)$ & 0.712 \\
\hline Class 2 (HLA-DR) & $1(1-1)$ & $1(0-2)$ & $1(0.5-1)$ & 0.749 & $1(1-1)$ & $1(0-2)$ & 0.683 \\
\hline CPRA & & & & 0.024 & & & 0.72 \\
\hline $0 \%$ & $39(86.7 \%)$ & $29(70.7 \%)$ & $4(50 \%)$ & & $54(75.0 \%)$ & $18(81.8 \%)$ & \\
\hline$<50 \%$ & $6(13.3 \%)$ & $8(19.5 \%)$ & $4(50 \%)$ & & $14(19.4 \%)$ & $4(18.2 \%)$ & \\
\hline$\geq 50 \%$ & $0(0 \%)$ & $4(9.8 \%)$ & $0(0 \%)$ & & $4(5.6 \%)$ & $0(0 \%)$ & \\
\hline Induction therapy regimen & & & & 1.0 & & & 1.0 \\
\hline Anti-thymocyte globulin & $5(11.1 \%)$ & $4(9.8 \%)$ & $1(12.5 \%)$ & & $8(11.1 \%)$ & $2(9.1 \%)$ & \\
\hline Basiliximab & 40 (88.9\%) & $37(90.2 \%)$ & $7(87.5 \%)$ & & $64(88.9 \%)$ & $20(90.9 \%)$ & \\
\hline Maintenance regimen immunosuppressants & & & & 0.118 & & & 0.84 \\
\hline $\mathrm{CsA}+\mathrm{MMF}+\mathrm{PD}$ & $10(22.2 \%)$ & $2(4.9 \%)$ & $1(12.5 \%)$ & & $11(15.3 \%)$ & $2(9.1 \%)$ & \\
\hline$F K+M M F+P D$ & $34(75.6 \%)$ & $38(92.7 \%)$ & $7(87.5 \%)$ & & $59(81.9 \%)$ & $20(90.9 \%)$ & \\
\hline Sirolimus or everolimus combination & $1(2.2 \%)$ & $1(2.4 \%)$ & $0(0 \%)$ & & $2(2.8 \%)$ & $0(0 \%)$ & \\
\hline \multicolumn{8}{|l|}{ AR } \\
\hline Within 1 month post-transplant & $8(17.8 \%)$ & $10(24.4 \%)$ & $0(0 \%)$ & 0.468 & $13(18.1 \%)$ & $5(22.7 \%)$ & 0.627 \\
\hline Within 3 months post-transplant & $8(17.8 \%)$ & $13(31.7 \%)$ & $1(12.5 \%)$ & 0.245 & $16(22.2 \%)$ & $6(27.3 \%)$ & 0.625 \\
\hline Within 6 months post-transplant & $9(20.0 \%)$ & $16(39.0 \%)$ & $2(25.0 \%)$ & 0.153 & $19(26.4 \%)$ & $8(36.4 \%)$ & 0.368 \\
\hline Within 12 months post-transplant & $10(22.2 \%)$ & $17(41.5 \%)$ & $2(25.0 \%)$ & 0.152 & $21(29.2 \%)$ & $8(36.4 \%)$ & 0.523 \\
\hline During F/U period & $17(37.8 \%)$ & $23(56.1 \%)$ & $3(37.5 \%)$ & 0.139 & $29(40.3 \%)$ & $14(63.6 \%)$ & 0.062 \\
\hline \multicolumn{8}{|l|}{ AR or MVI only } \\
\hline Within 1 month post-transplant & $9(20.0 \%)$ & $13(31.7 \%)$ & $0(0 \%)$ & 0.247 & $16(22.2 \%)$ & $6(27.3 \%)$ & 0.625 \\
\hline Within 3 months post-transplant & $11(24.4 \%)$ & $16(39.0 \%)$ & $1(12.5 \%)$ & 0.196 & $19(26.4 \%)$ & $9(40.9 \%)$ & 0.196 \\
\hline Within 6 months post-transplant & $12(26.7 \%)$ & $19(46.3 \%)$ & $2(25.0 \%)$ & 0.138 & $22(30.6 \%)$ & $11(50.0 \%)$ & 0.099 \\
\hline Within 12 months post-transplant & $13(28.9 \%)$ & $20(48.8 \%)$ & $2(25.0 \%)$ & 0.128 & $24(33.3 \%)$ & $11(50.0 \%)$ & 0.161 \\
\hline During F/U period & 20 (44.4\%) & $26(63.4 \%)$ & $3(37.5 \%)$ & 0.101 & $32(44.4 \%)$ & $17(77.3 \%)$ & 0.008 \\
\hline Pre-transplant anti-AT1R levels, U/mL & $6.26 \pm 2.2$ & $12.50 \pm 1.6$ & $20.72 \pm 3.1$ & $<0.001$ & $10.0 \pm 4.8$ & $11.0 \pm 5.1$ & 0.412 \\
\hline Pre-transplant AECA (+) & $10(22.2 \%)$ & $9(22.0 \%)$ & $3(37.5 \%)$ & 0.616 & & & \\
\hline
\end{tabular}

${ }^{*}$ Continuous variables are reported as mean \pm SD or median (interquartile range) and categorical variables are listed as total number (\%). KolmogorovSmirnov test was employed for test of normality assumption.

Abbreviations: ECXM, endothelial cell crossmatch; Anti-AT1R, anti-angiotensin II type 1 receptor antibodies; AECA, anti-endothelial cell antibodies; BMI, body mass index; cPRA, calculated panel reactive antibodies; CsA, cyclosporine A; MMF, mycophenolate mofetil; PD, prednisolone; FK, tacrolimus; AR, acute rejection; MVI, microvascular inflammation. 


\section{RESULTS}

Characteristics of the study population

Of the 94 KT recipients, 93 (98.9\%) had received their first kidney transplant and one (1.1\%) had received a second kidney transplant following primary graft failure due to renal vein thrombosis. The main demographic characteristics of the recipients are presented in Table 1. No graft failure occurred during the follow-up period ( $996 \pm 292$ days); however, one recipient (1.1\%) died owing to septic shock irrespective of an immunologic event. Nine of the 94 recipients (9.6\%) developed de novo HLA antibodies. Of the five recipients who experienced rejection episodes, three had pre-transplant anti-AT1R levels of 10-17 U/mL; how- ever, none had positive pre-transplant AECAs. There was no significant association between de novo HLA antibodies and rejection (AR, $P=0.555$; AR or MVI only, $P=0.392$ ).

\section{Correlation between clinical outcomes and anti-AT1R levels and AECA results}

The demographic characteristics of the recipients based on anti-AT1R levels and AECA using ECXM assay are presented in Table 2. No significant differences in anti-AT1R levels were found between AECA (-) and AECA (+) recipients. Additionally, there were no significant differences in the AECA $(+)$ rate among the three anti-AT1R groups as well as between the groups using the new optimal anti-AT1R level cut-off value, $11.5 \mathrm{U} / \mathrm{mL}$ (19.0\% in

Table 3. Risk factors associated with AR based on Cox proportional-hazards regression analysis

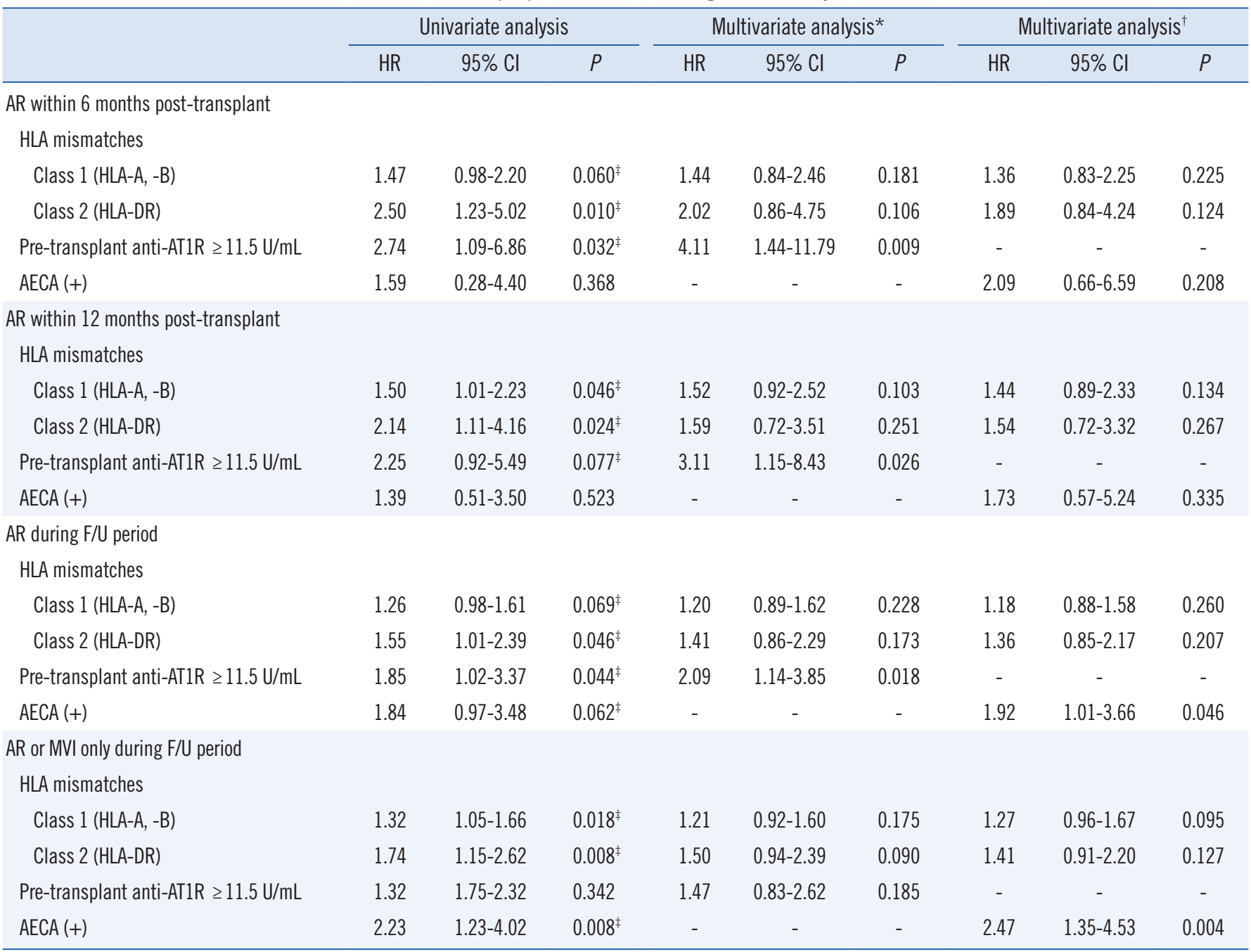

*The pre-transplant anti-AT1R $\geq 11.5 \mathrm{U} / \mathrm{mL}$ results were included as an independent variable; ${ }^{\dagger}$ The AECA result by ECXM assay was included as an independent variable; ${ }^{\ddagger} P<0.2$ was subjected to a backward stepwise Cox regression model (multivariate analysis).

Abbreviations: AR, acute rejection; HR, hazard ratio; Cl, confidence interval; Anti-AT1R, anti- angiotensin II type 1 receptor antibodies; AECA, anti-endothelial cell antibodies; MVI, microvascular inflammation; ECXM, endothelial cell crossmatch. 


\section{Yu S, et al.}

Pre-transplant anti-AT1R and AECA in low-risk KT

the anti-AT1R $<11.5 \mathrm{U} / \mathrm{mL}$ group vs. $44.0 \%$ in the anti-AT1R $>11.5 \mathrm{U} / \mathrm{mL}$ group, $P=0.218$ ). The prevalence of $A R$ within 12 months post-transplant did not differ significantly based on anti-AT1R levels and AECA results. However, when a cut-off value of $11.5 \mathrm{U} / \mathrm{mL}$ was applied, it independently predicted a higher risk for AR. AECA (+) recipients had a higher risk for AR or MVI only during the follow-up period (Table 3). Recipient characteristics based on combined immunologic status of pretransplant anti-AT1R levels and AECA using ECXM assay are shown in Table 4.

Kaplan-Meier analysis demonstrated that recipients with antiAT1R $\geq 11.5 \mathrm{U} / \mathrm{mL}$ had a higher risk of AR than those with antiAT1R $<11.5 \mathrm{U} / \mathrm{mL}, A E C A(+)$ recipients had a higher risk for AR or MVI only than AECA (-) recipients, and AECA (+) recipients

Table 4. Recipient characteristics and post-transplant outcomes according to combined immunologic status of pre-transplant anti-AT1R levels and AECA using ECXM assay

\begin{tabular}{|c|c|c|c|c|c|}
\hline & $\begin{array}{l}\text { Anti-AT1R }<11.5 \mathrm{U} / \mathrm{mL} \\
\text { and AECA }(-)(\mathrm{N}=47)^{*}\end{array}$ & $\begin{array}{l}\text { Anti-AT1R } \geq 11.5 \mathrm{U} / \mathrm{mL} \\
\text { and AECA }(-)(\mathrm{N}=25)^{*}\end{array}$ & $\begin{array}{l}\text { Anti-AT1R }<11.5 \mathrm{U} / \mathrm{mL} \\
\text { and AECA }(+)(\mathrm{N}=11)^{*}\end{array}$ & $\begin{array}{l}\text { Anti-AT1R } \geq 11.5 \mathrm{U} / \mathrm{mL} \\
\text { and AECA }(+)(\mathrm{N}=11)^{*}\end{array}$ & $P$ \\
\hline Age (yr) & $47.9 \pm 10.8$ & $47.4 \pm 12.3$ & $50.2 \pm 10.3$ & $40.8 \pm 13.0$ & 0.331 \\
\hline Gender, male & $30(63.8 \%)$ & $13(52.0 \%)$ & $9(81.8 \%)$ & $5(45.5 \%)$ & 0.242 \\
\hline BMI & $23.6 \pm 3.6$ & $23.3 \pm 4.7$ & $22.6 \pm 2.9$ & $20.6 \pm 2.8$ & 0.089 \\
\hline \multicolumn{6}{|l|}{ HLA mismatches } \\
\hline Class 1 (HLA-A,-B) & $2(1-3)$ & $2(1-3)$ & $3(2-3)$ & $2(1-2)$ & 0.666 \\
\hline Class 2 (HLA-DR) & $1(1-1)$ & $1(0-1)$ & $1(0-2)$ & $1(0-2)$ & 0.822 \\
\hline CPRA & & & & & 0.526 \\
\hline $0 \%$ & $37(78.7 \%)$ & $17(68.0 \%)$ & $10(90.9 \%)$ & $8(72.7 \%)$ & \\
\hline$<50 \%$ & $9(19.2 \%)$ & $5(20.0 \%)$ & $1(9.1 \%)$ & $3(27.3 \%)$ & \\
\hline$\geq 50 \%$ & $1(2.1 \%)$ & $3(12.0 \%)$ & $0(0 \%)$ & $0(0 \%)$ & \\
\hline Induction therapy regimen & & & & & 0.61 \\
\hline Anti-thymocyte globulin & $5(10.6 \%)$ & $3(12.0 \%)$ & 2 (18.2\%) & $0(0 \%)$ & \\
\hline Basiliximab & $42(89.4 \%)$ & $22(88.0 \%)$ & $9(81.8 \%)$ & $11(100 \%)$ & \\
\hline Maintenance regimen immunosuppressants & & & & & 0.704 \\
\hline $\mathrm{CsA}+\mathrm{MMF}+\mathrm{PD}$ & $8(17.0 \%)$ & $3(12.0 \%)$ & $2(18.2 \%)$ & $0(0 \%)$ & \\
\hline $\mathrm{FK}+\mathrm{MMF}+\mathrm{PD}$ & $37(78.7 \%)$ & $22(88.0 \%)$ & $9(81.8 \%)$ & $11(100 \%)$ & \\
\hline Sirolimus or everolimus combination & $2(4.3 \%)$ & $0(0 \%)$ & $0(0 \%)$ & $0(0 \%)$ & \\
\hline \multicolumn{6}{|l|}{ AR } \\
\hline Within 1 month post-transplant & $8(17.0 \%)$ & $5(20.0 \%)$ & $2(18.2 \%)$ & $3(27.3 \%)$ & 0.893 \\
\hline Within 3 months post-transplant & $8(17.0 \%)$ & $8(32.0 \%)$ & $3(27.3 \%)$ & $3(27.3 \%)$ & 0.526 \\
\hline Within 6 months post-transplant & $8(17.0 \%)$ & $11(44.0 \%)$ & $4(36.4 \%)$ & $4(36.4 \%)$ & 0.099 \\
\hline Within 12 months post-transplant & $10(21.3 \%)$ & $11(44.0 \%)$ & $4(36.4 \%)$ & $4(36.4 \%)$ & 0.238 \\
\hline During F/U period & $15(31.9 \%)$ & $14(56.0 \%)$ & $7(63.6 \%)$ & $7(63.6 \%)$ & 0.071 \\
\hline \multicolumn{6}{|l|}{ AR or MVI only } \\
\hline Within 1 month post-transplant & $11(23.4 \%)$ & $5(20.0 \%)$ & $3(27.3 \%)$ & $3(27.3 \%)$ & 0.952 \\
\hline Within 3 months post-transplant & $11(23.4 \%)$ & $8(32.0 \%)$ & $6(54.5 \%)$ & $3(27.3 \%)$ & 0.268 \\
\hline Within 6 months post-transplant & $11(23.4 \%)$ & $11(44.0 \%)$ & $7(63.6 \%)$ & $4(36.4 \%)$ & 0.069 \\
\hline Within 12 months post-transplant & $13(27.7 \%)$ & $11(44.0 \%)$ & $7(63.6 \%)$ & $4(36.4 \%)$ & 0.152 \\
\hline During F/U period & $18(38.3 \%)$ & $14(56.0 \%)$ & $10(90.9 \%)$ & $7(63.6 \%)$ & 0.012 \\
\hline
\end{tabular}

${ }^{*}$ Continuous variables are reported as mean \pm SD or median (interquartile range), and categorical variables are listed as number (\%). Kolmogorov-Smirnov test was employed for testing normality assumption.

Abbreviations: Anti-AT1R, anti-angiotensin II type 1 receptor antibodies; AECA, anti-endothelial cell antibodies; ECXM, endothelial cell crossmatch; BMI, body mass index; CPRA, calculated panel reactive antibodies; CsA, cyclosporine A; MMF, mycophenolate mofetil; PD, prednisolone; FK, tacrolimus; AR, acute rejection; MVI, microvascular inflammation. 
A

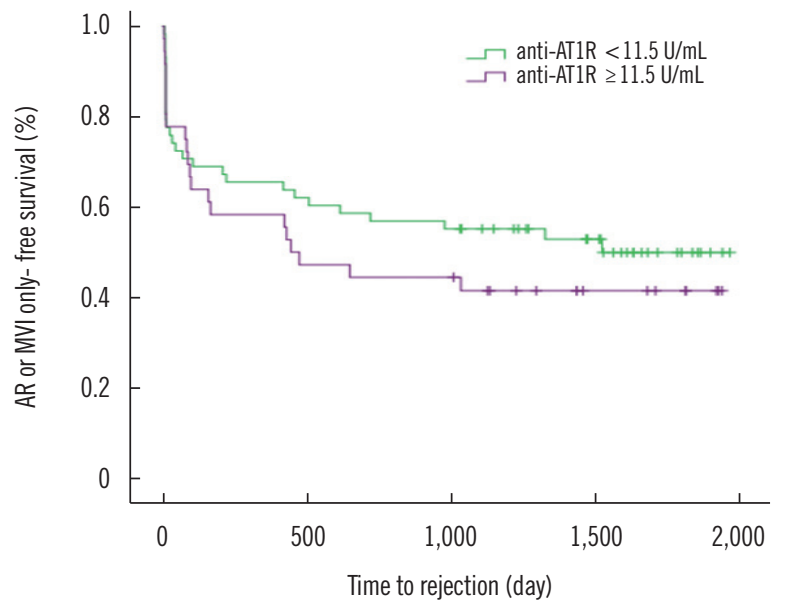

C

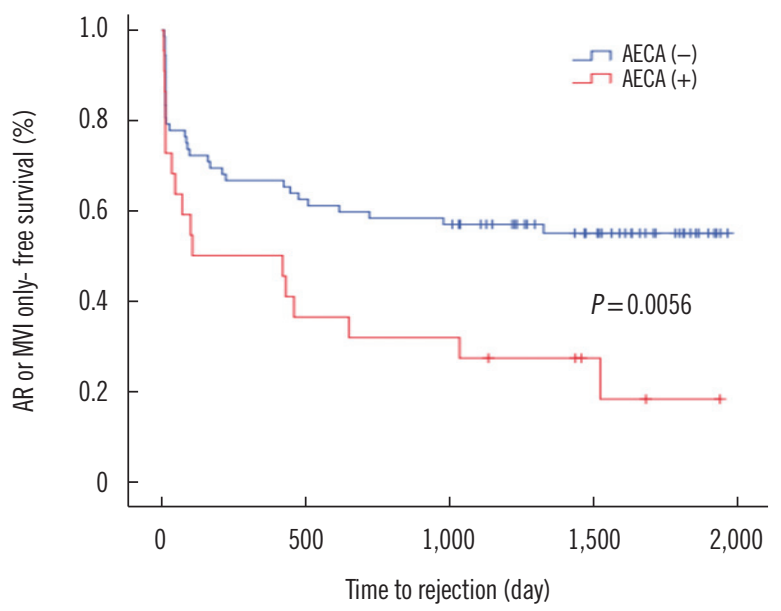

E

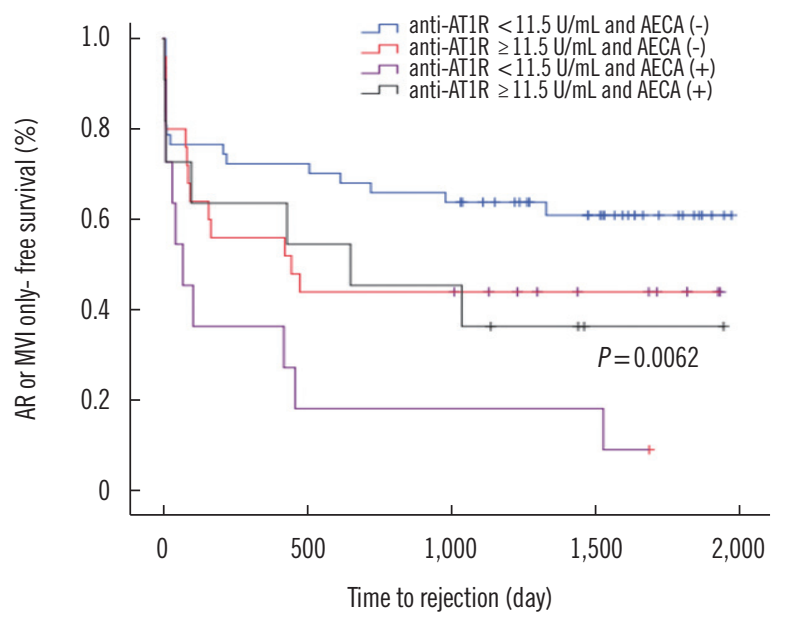

B

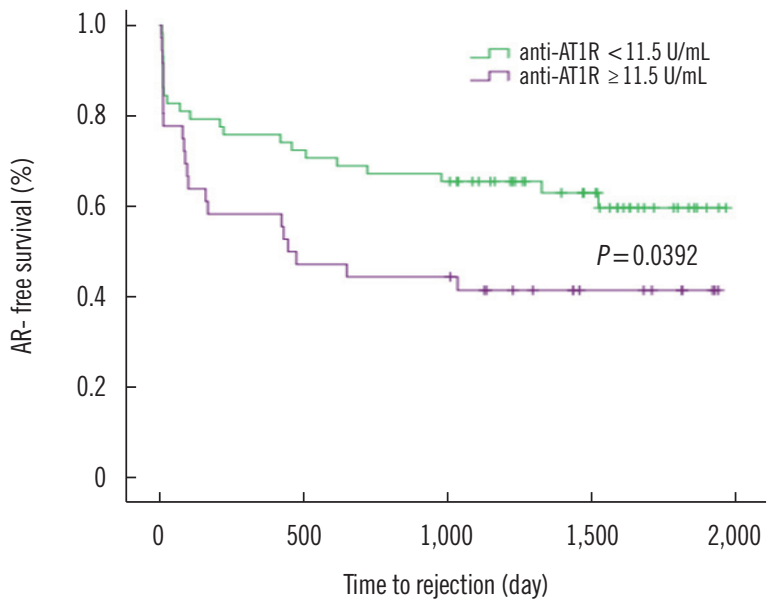

D

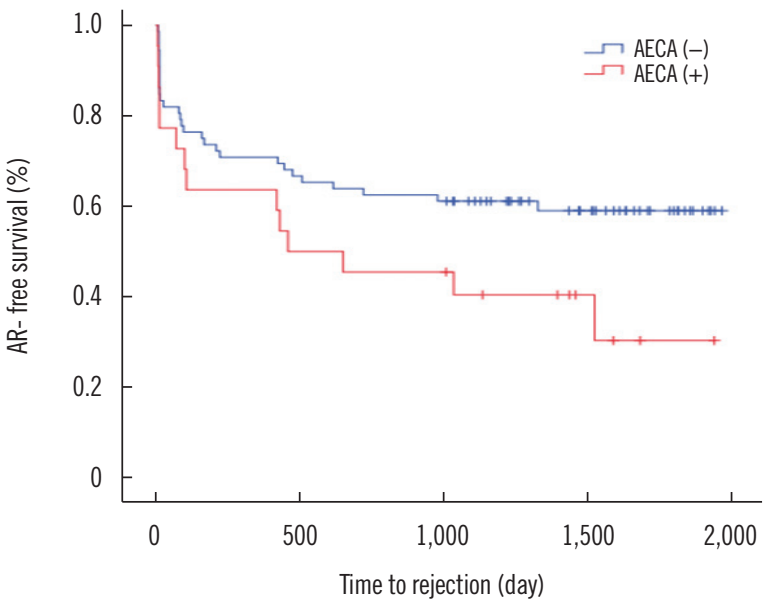

F

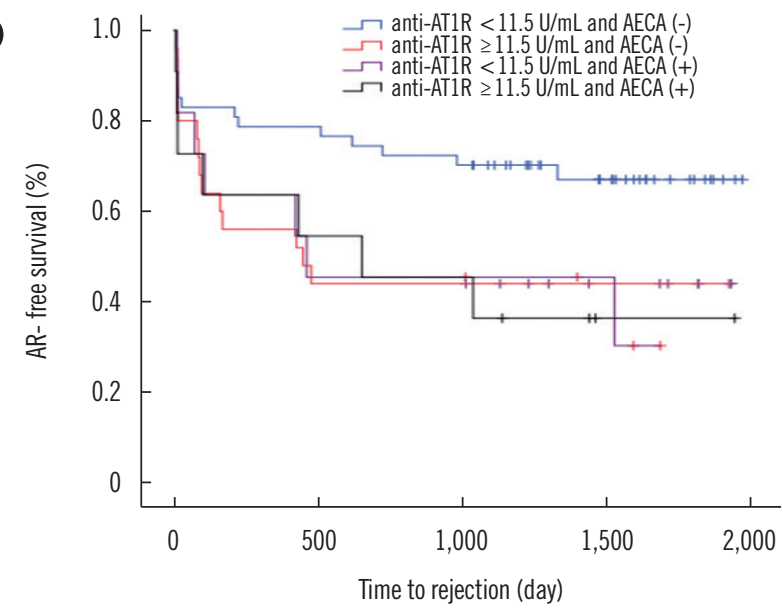

Fig. 1. Clinical outcomes according to the anti-AT1R levels and AECA status using ECXM assay. (A) No significant differences in AR or MVI only free survival rates are seen between recipients with anti-AT1R $\geq 11.5 \mathrm{U} / \mathrm{mL}(\mathrm{N}=36)$ and those with anti-AT1R $<11.5 \mathrm{U} / \mathrm{mL}(\mathrm{N}=58)$. (B) Recipients with anti-AT1R $\geq 11.5 \mathrm{U} / \mathrm{mL}$ have a higher risk of AR than those with anti-AT1R $<11.5 \mathrm{U} / \mathrm{mL}(P=0.039)$. (C) AECA (+) recipients ( $N=22)$ have a higher risk of AR or MVI only than AECA $(-)$ recipients $(N=72)(P=0.006)$; (D) There is no significant difference in the AR free survival rates. (E) AECA (+) recipients with anti-AT1R $<11.5 \mathrm{U} / \mathrm{mL}(\mathrm{N}=11)$ have a higher risk of AR or MVI only than other recipients $(P=0.006) ;(F)$ There are no significant differences in AR free survival rates among the four groups.

Abbreviations: Anti-AT1R, anti-angiotensin II type 1 receptor antibodies; AECA, anti-endothelial cell antibodies; ECXM, endothelial cell crossmatch; AR, acute rejection; MVI, microvascular inflammation. 
A

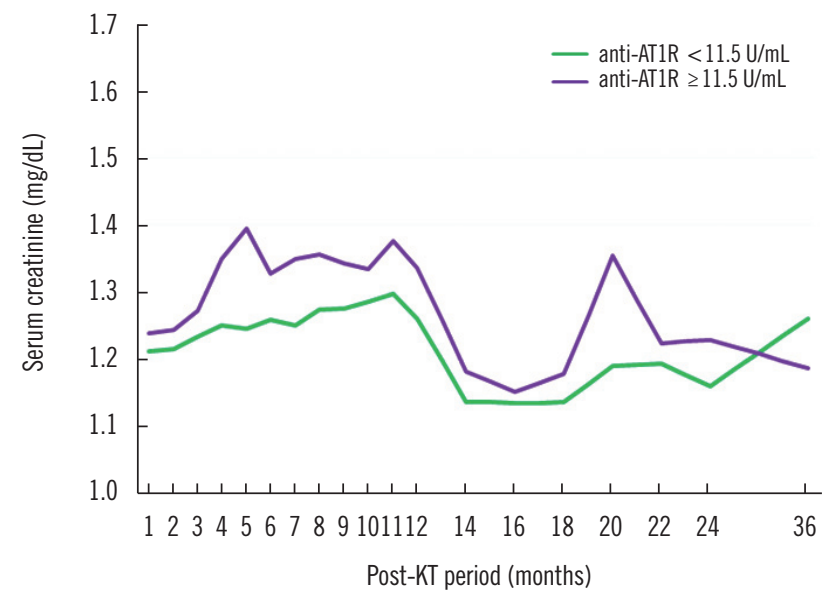

C

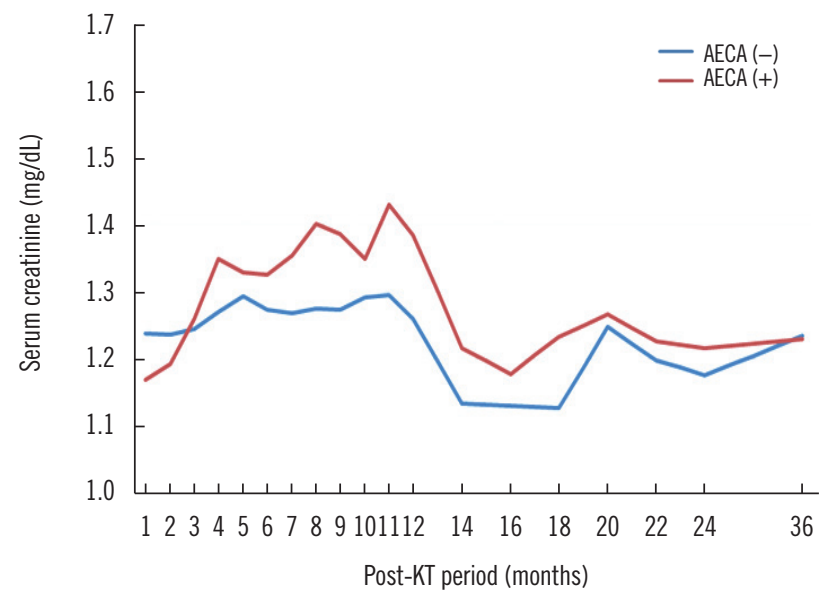

E

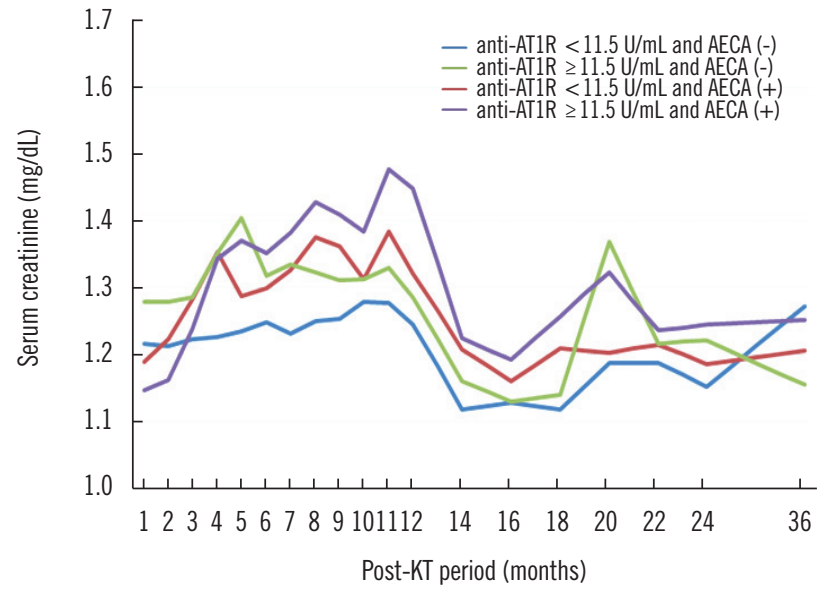

B

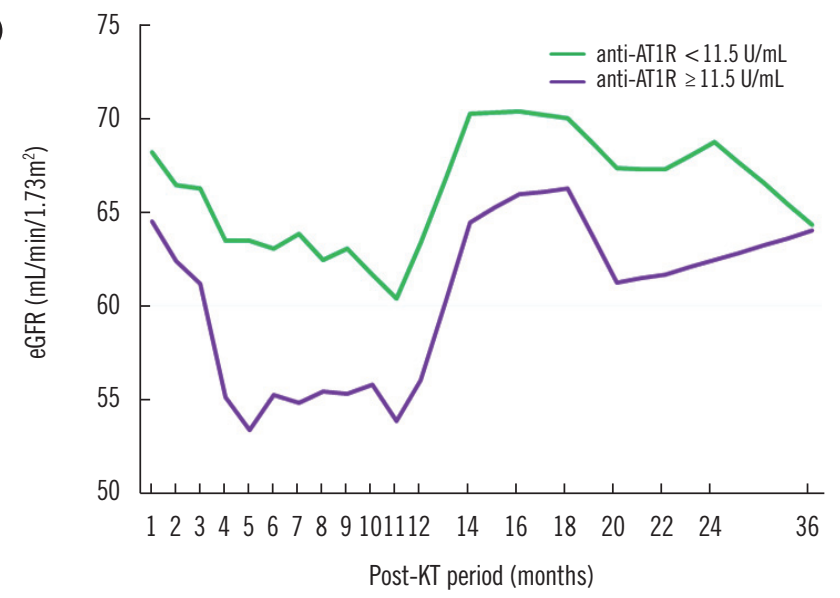

D

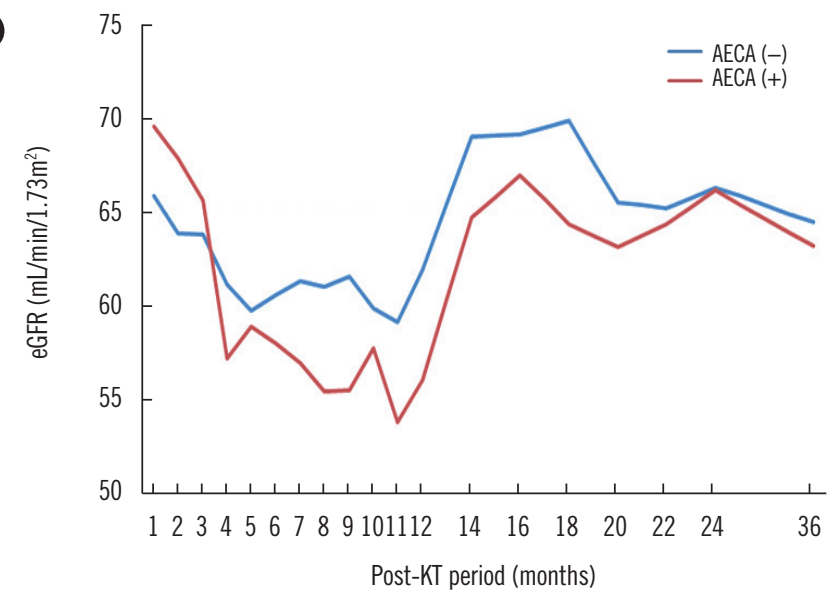

$\boldsymbol{F}$

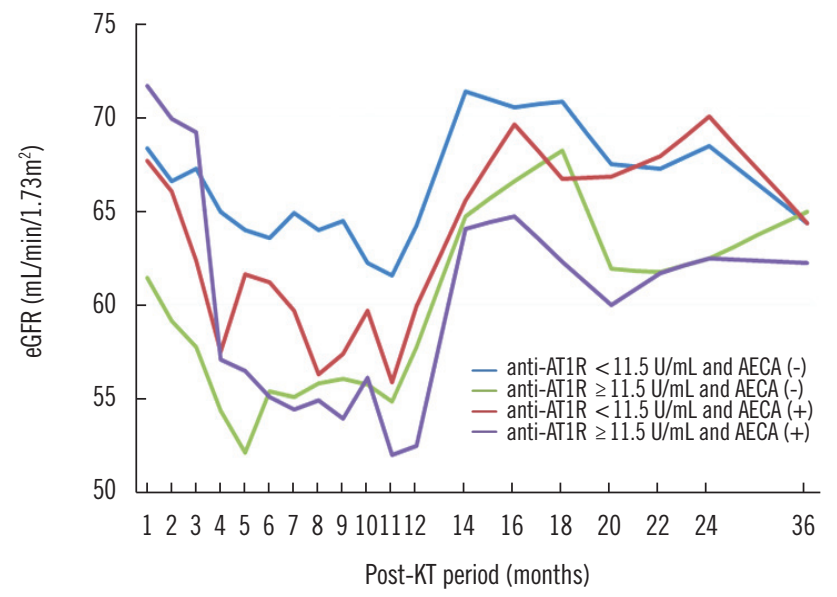

Fig. 2. Effect of anti-AT1R levels and AECA status using ECXM assay on renal function during the post-KT period. Recipients with pretransplant anti-AT1R $\geq 11.5 \mathrm{U} / \mathrm{mL}$ show significantly lower eGFR (B) but not creatinine levels (A) at 6 and 12 months post $\mathrm{KT}(P=0.012$; $P=0.012$, respectively), compared with those at one month post-KT. AECA $(+)$ recipients have significantly higher creatinine levels $(C)$ and lower eGFRs (D) at six $(P=0.003 ; P=0.028$, respectively) and 12 months $(P<0.001 ; P=0.011$, respectively), compared with those at one month post-KT. The change in the pattern of creatinine levels in AECA (+) recipients from one to 12 months post-KT is significantly different compared with that in AECA (-) recipients $(P=0.038)(C)$. AECA (+) recipients with anti-AT1R $\geq 11.5 \mathrm{U} / \mathrm{mL}$ show significantly different changes in the pattern of creatinine levels (E) from one to 12 months post-KT $(P=0.045)$ compared with other recipients, and significantly higher creatinine levels and lower eGFRs $(F)$ at 12 months $(P<0.001 ; P=0.028)$ compared with those at one month post-KT.

Abbreviations: Anti-AT1R, anti-angiotensin II type 1 receptor antibodies; AECA, anti-endothelial cell antibodies; ECXM, endothelial cell crossmatch; KT, kidney transplantation; eGFR, estimated glomerular filtration rate; MVI, microvascular inflammation. 
with anti-AT1R $<11.5 \mathrm{U} / \mathrm{mL}$ had a higher risk of AR or MVI only than other recipients (Fig. 1). Based on multivariate analysis, pre-transplant AECA (-) recipients with anti-AT1R $\geq 11.5 \mathrm{U} / \mathrm{mL}$ had a higher risk of AR within six months post-transplant (HR 5.68; $P=0.018$ ), and pre-transplant AECA (+) recipients with anti-AT1R $<11.5 \mathrm{U} / \mathrm{mL}$ had a higher risk of AR or MVI only within 6 months post-transplant (HR 7.37; $P=0.037$ ) and during the follow-up period (HR 4.14; $P=0.002$ ).

We measured anti-AT1R levels in the sera of 29 BPAR recipients at the time of kidney biopsy. Only two recipients had an anti-AT1R $\geq 11.5 \mathrm{U} / \mathrm{mL}$ at the time of AR and were among the 12 recipients who had an anti-AT1R $\geq 11.5 \mathrm{U} / \mathrm{mL}$ pre-KT. Seventeen recipients had an anti-AT1R $<11.5 \mathrm{U} / \mathrm{mL}$ both pre-KT and at the time of AR. The median pre-transplant anti-AT1R level of these 29 recipients was $11.2 \mathrm{U} / \mathrm{mL}$ (range: 3.8-25.4 U/mL), which was higher than that at the time of $A R(7.9 \mathrm{U} / \mathrm{mL}$; range, 4.4-12.7 U/mL; $P=0.029$ ).

There was no correlation between pre-transplant anti-AT1R levels and post-transplant creatinine levels; however, recipients with pre-transplant anti-AT1R $\geq 11.5 \mathrm{U} / \mathrm{mL}$ had a significantly lower eGFR at both six and 12 months post-transplant $(P=0.012$; $P=0.020$ ) compared with that at one month post-transplant (Fig. $2 \mathrm{~A}$ and $\mathrm{B}$ ). Additionally, recipients with anti-AT1R $\geq 11.5 \mathrm{U} / \mathrm{mL}$ showed significantly lower eGFR for six months post-transplant compared with recipients with pre-transplant anti-AT1R $<11.5$ $\mathrm{U} / \mathrm{mL}(P=0.010)$, which continued until 24 months post-transplant. Renal function, estimated using post-transplant creatinine and eGFR, differed depending on AECA results. AECA (+) recipients showed a rapid increase in creatinine and decrease in eGFR at approximately three months; after this point, they showed persistently higher creatinine and lower eGFR until 20 months (Fig. 2C and D). Interestingly, pre-transplant AECA (+) recipients with anti-AT1R $\geq 11.5 \mathrm{U} / \mathrm{mL}$ were not associated with AR or MVI only at any time point during the entire follow-up period; however, they had significantly different changes in the creatinine level pattern from one to 12 months post-transplant $(P=0.045)$, as well as significantly higher creatinine and a lower eGFR at 12 months $(P<0.001 ; P=0.028)$ compared with levels at one month post-transplant (Fig. 2E and F).

\section{DISCUSSION}

Our aim was to evaluate the impact of pre-transplant anti-AT1R and AECA on post-transplant outcomes in low-risk LDKT recipients. The target antigen for AECA detected in the ECXM assay is unknown; thus, all antigens expressed on endothelial cells are possible candidates [4]. Previous studies have reported that ECXM assay can detect anti-AT1R as well as antibodies against targets other than AT1R [11-13]. Philogene, et al. [12, 13] reported that recipients who were positive for AECA have higher anti-AT1R levels; however, we found that there was no correlation between pre-transplant anti-AT1R levels and AECA status, and that $\mathrm{KT}$ outcomes are affected differently by pre-transplant anti-AT1R levels and AECA status. The presence of pre-transplant anti-AT1R was a significant risk factor for the development of $A R$, whereas AECA status was associated with post-transplant renal function, estimated using creatinine levels or eGFR and $\mathrm{AR}$ or MVI only. MVI, which was included as a sign of ABMR in the revised Banff 2017 classification [18], can be observed not only in ABMR but also in acute tubular necrosis, glomerulonephritis, and acute T-cell-mediated rejection [19, 20]. However, an MVI $\geq 2$ is significantly associated with a histological diagnosis of acute and chronic ABMR [21]. Therefore, the progression to ABMR should be carefully monitored in recipients with MVI only who are not yet BPAR-compatible.

Recent studies have drawn very diverse conclusions regarding the effect of pre-transplant anti-AT1R on KT outcomes [16, $24,25]$. We identified an optimal cut-off value for anti-AT1R of $11.5 \mathrm{U} / \mathrm{mL}$, which independently predicted a higher risk of AR in low risk LDKT recipients within six and 12 months post-transplant. We hypothesize that these AR episodes contributed to the decreased eGFR in the early stages of KT (Fig. 2B). The difference in eGFR between anti-AT1R $\geq 11.5$ and $<11.5 \mathrm{U} / \mathrm{mL}$ is offset, most likely by proper management for increased creatinine and AR episodes. However, recipients with anti-AT1R $\geq 11.5 \mathrm{U} / \mathrm{mL}$ appear to have persistently lower eGFR compared with those with $<11.5 \mathrm{U} / \mathrm{mL}$ until 36 months. AECA (+) recipients demonstrated decreased eGFR at approximately three months and maintained lower eGFR than AECA (-) recipients until 24 months, regardless of AR episodes. Although it remains unclear why eGFR decreases and serum creatinine increases regardless of AR in AECA (+) recipients, we have observed that unlike AT1R, an AECA (+) result was significantly associated not only with AR but also with MVI only during the follow-up period. A previous study has reported that endothelin-1 type A receptor antibody, one of the AECA candidates, reduces renal function and increases intimal arteritis post-transplant [24]. Long term follow-up is needed to clarify the effect of anti-AT1R and AECA status on graft outcome.

Several studies have explored an association between HLADSA and anti-AT1R [7, 12, 27]. A few studies have shown that recipients with both pre-transplant HLA-DSA and anti-AT1R had 
lower graft survival rates compared with recipients with either one, suggesting that there is a synergistic effect between pretransplant HLA-DSA and anti-AT1R [7, 12]. Determining the mechanism by which anti-AT1R affect KT outcome is difficult, as pre-transplant HLA-DSA have a greater effect on KT outcome $[14,15]$. Thus, we enrolled low-risk LDKT recipients, without pre-transplant HLA-DSA, in order to exclude the possibility that HLA-DSA affects KT outcomes. Several previous studies have reported that the de novo development of HLA-DSA is significantly higher in recipients with positive anti-AT1R [7, 24, 27]. We identified six recipients (6.4\%) who developed de novo HLA-DSA; however, none were positive for AECA or anti-AT1R ( $>17 \mathrm{U} / \mathrm{mL}$ ). This may suggest that the development of de novo HLA-DSA does not require pre-existing AECA or anti-AT1R. We did not observe a correlation between de novo HLA-DSA and $A R$, probably because of the small number of recipients who developed de novo HLA-DSA. This suggests that the presence of pre-transplant anti-AT1R and AECA have a greater effect on rejection risk and graft function than de novo HLA-DSA in lowrisk LDKT recipients. In our study, the renal function of the recipients tended to improve after 12 months post-transplant (Fig. 2); this might be due to the prospective management employed according to the biopsy findings in the 73 recipients who underwent the one-yr protocol biopsy. Recipients under subclinical rejection were treated with steroid pulse therapy. In the cases of tacrolimus toxicity or BK virus-associated nephritis, the immunosuppressant was replaced with a lower intensity immunosuppressant such as sirolimus.

The limitation of this study is that we evaluated pre-transplant anti-AT1R levels and AECA status in all recipients, except for the post-transplant anti-AT1R levels in 29 recipients who experienced a biopsy proven rejection. Thus, we cannot exclude the impact of post-transplant anti-AT1R and AECA on transplant outcomes. However, except for two recipients with BPAR, the anti-AT1R levels at the time of rejection were $<11.5 \mathrm{U} / \mathrm{mL}$. This is consistent with previous study demonstrating that the antiAT1R levels at the time of rejection were lower than the pretransplant levels in most recipients with rejection episodes [8], probably owing to absorption of anti-AT1R to the graft.

In conclusion, we found that the presence of pre-transplant anti-ATIR and AECA, has a significant impact on the posttransplant outcomes in low-risk LDKT recipients. Pre-transplant anti-AT1R level was a significant risk factor for the development of $A R$, while AECA status was associated with impaired renal function regardless of AR. Therefore, evaluation of anti-AT1R levels and AECA before KT would be necessary to stratify the risk of graft dysfunction and predict the risk of AR due to nonHLA antibodies, particularly in a low-risk LDKT setting.

\section{ACKNOWLEDGEMENTS}

We wish to thank to Bok Nyeo Kim, R.N. for coordinating recipient enrollment and Jung Hae Kim, M.T. for his excellent technical support in performing the assays.

\section{AUTHOR CONTRIBUTIONS}

SY collected and analyzed the data and wrote the paper; $\mathrm{HJH}$ designed the study; KWL, JBP, SJK, WH, HRJ, GYK, and HHM performed the study; and ESK designed and performed the study and wrote the paper.

\section{CONFLICT OF INTEREST}

No potential conflicts of interest relevant to this paper were reported

\section{RESEARCH FUNDING}

None declared.

\section{ORCID}

Shinae Yu https://orcid.org/0000-0002-9527-5853

Hee Jae Huh Kyo Won Lee Jae Berm Park Sung-Joo Kim Wooseong Huh Hye Ryoun Jang Ghee Young Kwon Hyung Hwan Moon Eun-Suk Kang https://orcid.org/0000-0001-8999-7561 https://orcid.org/0000-0002-2722-7817 https://orcid.org/0000-0001-9117-2278 https://orcid.org/0000-0001-9251-1673 https://orcid.org/0000-0001-8174-5028 https://orcid.org/0000-0001-9856-6341 https://orcid.org/0000-0002-5551-4178 https://orcid.org/0000-0002-4212-4568 https://orcid.org/0000-0001-6386-6520

\section{REFERENCES}

1. Terasaki PI. Humoral theory of transplantation. Am J Transplant 2003;3: 665-73.

2. Opelz G and Collaborative Transplant Study. Non-HLA transplantation immunity revealed by lymphocytotoxic antibodies. Lancet 2005;365: 1570-6.

3. Han F, Lv R, Jin J, Wu J, Chen Y, Wang H, et al. Pre-transplant serum concentrations of anti-endothelial cell antibody in panel reactive antibody negative renal recipients and its impact on acute rejection. Clin 
Chem Lab Med 2009;47:1265-9.

4. Breimer ME, Rydberg L, Jackson AM, Lucas DP, Zachary AA, Melancon $\mathrm{JK}$, et al. Multicenter evaluation of a novel endothelial cell crossmatch test in kidney transplantation. Transplantation 2009;87:549-56.

5. Xavier P, Aires P, Sampaio S, Mendes C, Monteiro M, Alves H, et al. $\mathrm{XM}-\mathrm{ONE}$ detection of endothelium cell antibodies identifies a subgroup of HLA-antibody negative patients undergoing acute rejection. Transplant Proc 2011;43:91-4.

6. Ronda C, Borba SC, Ferreira SC, Glotz D, lanhez LE, Rodrigues H, et al. Non-human leukocyte antigen antibodies reactive with endothelial cells could be involved in early loss of renal allografts. Transplant Proc 2011; 43:1345-8.

7. Taniguchi M, Rebellato LM, Cai J, Hopfield J, Briley KP, Haisch CE, et al. Higher risk of kidney graft failure in the presence of anti-angiotensin Il type-1 receptor antibodies. Am J Transplant 2013;13:2577-89.

8. Giral M, Foucher Y, Dufay A, Van Huyen JP, Renaudin K, Moreau A, et al. Pretransplant sensitization against angiotensin II type 1 receptor is a risk factor for acute rejection and graft loss. Am J Transplant 2013;13: 2567-76.

9. Banasik M, Boratyńska M, Kościelska-Kasprzak K, Kamińska D, Bartoszek D, Zabińska M, et al. The influence of non-HLA antibodies directed against angiotensin II type 1 receptor (AT1R) on early renal transplant outcomes. Transpl Int 2014;27:1029-38.

10. Banasik M, Boratyńska M, Kościelska-Kasprzak K, Kamińska D, Zmonarski S, Mazanowska O, et al. Non-HLA antibodies: angiotensin II type 1 receptor (anti-AT1R) and endothelin-1 type A receptor (anti-ETAR) are associated with renal allograft injury and graft loss. Transplant Proc 2014; 46:2618-21.

11. Jackson AM, Sigdel TK, Delville M, Hsieh SC, Dai H, Bagnasco S, et al. Endothelial cell antibodies associated with novel targets and increased rejection. J Am Soc Nephrol 2015;26:1161-71.

12. Philogene MC, Bagnasco S, Kraus ES, Montgomery RA, Dragun D, Leffell MS, et al. Anti-angiotensin II Type 1 receptor and anti-endothelial cell antibodies: A cross-sectional analysis of pathological findings in allograft biopsies. Transplantation 2017;101:608-15.

13. Philogene MC, Zhou S, Lonze BE, Bagnasco S, Alasfar S, Montgomery RA, et al. Pre-transplant screening for non-HLA antibodies: who should be tested? Hum Immunol 2018;79:195-202.

14. Piotti G, Palmisano A, Maggiore U, Buzio C. Vascular endothelium as a target of immune response in renal transplant rejection. Front Immunol 2014;5:505.

15. Fuss A, Hope CM, Deayton S, Bennett GD, Holdsworth R, Carroll RP, et al. C4d-negative antibody-mediated rejection with high anti-angiotensin II type I receptor antibodies in absence of donor-specific antibodies. Nephrology (Carlton) 2015;20:467-73.
16. Min JW, Lee H, Choi BS, Park CW, Yang CW, Kim YS, et al. Clinical impact of pre-transplant antibodies against angiotensin II Type I receptor and major histocompatibility complex class I-related chain A in kidney transplant patients. Ann Lab Med 2018;38:450-7.

17. Reinsmoen NL, Lai CH, Mirocha J, Cao K, Ong G, Naim M, et al. Increased negative impact of donor HLA-specific together with non-HLAspecific antibodies on graft outcome. Transplantation 2014;97:595-601.

18. Haas M, Loupy A, Lefaucheur C, Roufosse C, Glotz D, Seron D, et al. The Banff 2017 Kidney Meeting Report: revised diagnostic criteria for chronic active $T$ cell-mediated rejection, antibody-mediated rejection, and prospects for integrative endpoints for next-generation clinical trials. Am J Transplant 2018;18:293-307.

19. Batal I, Girnita A, Zeevi A, Saab BA, Stockhausen S, Shapiro R, et al. Clinical significance of the distribution of C4d deposits in different anatomic compartments of the allograft kidney. Mod Pathol 2008;21:1490-8.

20. Gibson IW, Gwinner W, Bröcker V, Sis B, Riopel J, Roberts IS, et al. Peritubular capillaritis in renal allografts: prevalence, scoring system, reproducibility and clinicopathological correlates. Am J Transplant 2008;8: 819-25.

21. Gupta A, Broin PÓ, Bao Y, Pullman J, Kamal L, Ajaimy M, et al. Clinical and molecular significance of microvascular inflammation in transplant kidney biopsies. Kidney Int 2016;89:217-25.

22. Daniel V, Sadeghi M, Suesal C, Scherer S, Tran H, Gombos P, et al. Clinical relevance of preformed IgG and IgM antibodies against donor endothelial progenitor cells in recipients of living donor kidney grafts. Clin Transplant 2016;30:124-30.

23. Zitzner JR, Shah S, Jie C, Wegner W, Tambur AR, Friedewald JJ. A prospective study evaluating the role of donor-specific anti-endothelial crossmatch (XM-ONE assay) in predicting living donor kidney transplant outcome. Hum Immunol 2013;74:1431-6.

24. Gareau AJ, Wiebe C, Pochinco D, Gibson IW, Ho J, Rush DN, et al. Pretransplant AT1R antibodies correlate with early allograft rejection. Transpl Immunol 2018;46:29-35.

25. In JW, Park H, Rho EY, Shin S, Park KU, Park MH et al. Anti-angiotensin type 1 receptor antibodies associated with antibody-mediated rejection in patients without preformed HLA-donor-specific antibody. Transplant Proc 2014;46:3371-4.

26. Banasik M, Boratyńska M, Kościelska-Kasprzak K, Krajewska M, Mazanowska O, Kamińska D, et al. The impact of non-HLA antibodies directed against endothelin-1 type A receptors (ETAR) on early renal transplant outcomes. Transpl Immunol 2014;30:24-9.

27. Cuevas E, Arreola-Guerra JM, Hernández-Méndez EA, Salcedo I, Castelán N, Uribe-Uribe NO, et al. Pretransplant angiotensin II type 1-receptor antibodies are a risk factor for earlier detection of de novo HLA donor-specific antibodies. Nephrol Dial Transplant 2016;31:1738-45. 